\title{
Reactions to clinical reinterpretation of a gene variant by participants in a sequencing study
}

\author{
Jennifer M. Taber, $\mathrm{PhD}^{1}$, William M.P. Klein, $\mathrm{PhD}^{2}$, Katie L. Lewis, ScM${ }^{3}$, Jennifer J. Johnston, $\mathrm{PhD}^{3}$, \\ Leslie G. Biesecker, $\mathrm{MD}^{3}$ and Barbara B. Biesecker, PhD, $\mathrm{CGC}^{3}$
}

Purpose: As genome science advances, people receiving personalized genetic information may receive reinterpretations of pathogenicity. Little is known about responses to adjusted results. We examined how reinterpretations might affect attitudes about genetic testing and intentions to share results with family.

Methods: Data were collected from high-socioeconomic-status participants $(n=58)$ in a genome sequencing study. Twenty-nine originally learned they were carriers of Duarte variant galactosemia, based on a variant that was reclassified as benign. Positive testers $(n=19)$ had a newly identified causative variant and remained carriers. Negative testers $(n=10)$ learned they were no longer carriers. Twenty-nine controls were carriers for a disease of comparable severity with no reclassification. Participants completed baseline, immediate, and 3-month follow-up surveys.

Results: Approximately $80 \%$ of participants demonstrated complete or partially accurate recall of their results and reported positive or neutral reactions to their result and about genetic information more generally. Positive testers reported lower intentions to share the change in their result with family. Controls reported the lowest intentions to learn future results. There were no significant group differences or changes over time in perceived ambiguity or negative emotions.

Conclusion: The results suggest that high-socioeconomic-status participants understand reinterpretations conferring a neutral change or a change from carrier to noncarrier status. Participants' responses to changes in carrier results for a low-risk condition indicated minimal adverse effects.

Genet Med advance online publication 3 August 2017

Key Words: genetic testing; genome sequencing; reclassification; reinterpretation; return of results

\section{INTRODUCTION}

As new genomic discoveries are made, clinicians will increasingly inform people of variants that confer disease susceptibility or disease risk for family members. Interpreting information obtained through clinical genome and exome sequencing is complex, as is determining how to communicate this information to patients. ${ }^{1}$ People who undergo sequencing could have multiple subsequent meetings with their medical team, because the evidence base and interpretation standards are evolving. ${ }^{1}$ There is debate about which variants are actionable and should be returned to patients. ${ }^{2}$ Importantly, as evidence and standards evolve, patients may receive reevaluations of the pathogenicity of variants. Little is known about how people respond to these changes. The goal of this exploratory study was to examine how participants reacted to learning of a change regarding previously returned genetic risk information about carrier status for an autosomal recessive condition. Participants who previously received carrier results learned that one result was reclassified and they were now (i) a carrier of the same condition but based on a different mutation, or (ii) no longer a carrier.

Anxiety experienced by people with carrier status generally dissipates within 6 months, ${ }^{3}$ and even people at highly elevated genetic risk of breast and ovarian cancer generally do not report elevated distress. ${ }^{4}$ Our interest was responses to changes in carrier results, rather than to results themselves. In the present study, the reclassification was "neutral" news for most participants; none learned of increased risk. Indeed, people learning they were no longer carriers could respond positively. It was possible, however, that reinterpretation would diminish participants' trust in their results, in the study team, and/or in genetic information more broadly. Decreased trust could manifest as lower intentions to seek genetic information in the future, as focus group participants expected that genetic results returned to them would be well understood and accurately interpreted by the researchers. ${ }^{5}$ Conversely, a subset of participants from the parent project for the present study expected ambiguous and uncertain results from sequencing. ${ }^{6}$ Thus, we did not predict negative consequences from learning about a minor change in a sequencing result.

We could explore these issues because of a change in interpretation of a Duarte variant galactosemia, a metabolic disorder inherited in an autosomal recessive pattern. Participants' original reports described the c.940A $>\mathrm{G}$, p.Asn314Asp variant in GALT as pathogenic. It was later

${ }^{1}$ Department of Psychological Sciences, Kent State University, Kent, Ohio, USA; ${ }^{2}$ Behavioral Research Program, National Cancer Institute, Bethesda, Maryland, USA; ${ }^{3}$ National Human Genome Research Institute, Bethesda, Maryland, USA. Correspondence: Jennifer M. Taber (jtaber1@kent.edu)

Submitted 12 December 2016; accepted 8 May 2017; advance online publication 3 August 2017. doi:10.1038/gim.2017.88 
reclassified as benign; the variant was in linkage disequilibrium with the actual pathogenic variant, c.-119_-116del. We collected quantitative and qualitative data on responses to (i) the reclassification or (ii) receipt of carrier results that were not reclassified, including intention to share results with one's family and to learn additional genetic information, perceived accuracy and utility of results, negative emotional responses, and perceived ambiguity of results.

\section{MATERIALS AND METHODS \\ Participants and procedure}

Figure 1 shows the study design. Data were collected from participants enrolled in ClinSeq, an NIH study piloting the use of genome sequencing. ${ }^{7}$ Participants, aged 45-65 years, were recruited from the Washington, DC area. The National Human Genome Research Institute's institutional review board approved the study. Of the 998 ClinSeq participants, 551 who were enrolled for $>1$ month and had not yet received results completed a baseline survey. ${ }^{8}$ This survey provided baseline data for the present manuscript. All participants provided informed consent and blanket consent to receiving results over time. Most results would be received by choice, but participants were told that results with significant medical implications would be returned to them. Variants are identified through ancillary research projects or through periodic interrogation for variants that demonstrate clinical utility. Some participants received results emanating from targeted analyses. Participants have received variants conveying risk for coronary artery disease, secondary findings, and carrier results. Because this is a longitudinal investigation into clinical sequencing, participants will continue to be offered results. ${ }^{9}$

We refer in this paper to three studies that involved ClinSeq participants: (i) the "parent" study previously described, (ii) a randomized control trial (RCT) comparing different education modalities used to return carrier variants, and (iii) the present "reclassification" study. Reports returned through the RCT could contain any variants with a possibility of pathogenicity based on interrogating carrier status for $>1,300$ genes. As part of the RCT, 33 participants heterozygous for the c.940A > G (rs 2070074) variant in the GALT gene were informed that they were carriers for Duarte variant galactosemia. The information was given in person by a genetic counselor or through a Web platform (seven participants who received Duarte variant results outside of the RCT were excluded from the reclassification study). No follow-up surveys for the reclassification study were administered immediately after initial carrier results were returned (Figure 1). Duarte variant galactosemia is milder than other forms of galactosemia-affected newborns and children have few or no symptoms. ${ }^{10}$ Frequency of the Duarte variant is 5\% in the US population (monoallelic), consistent with the rate in our data. ${ }^{11}$

We developed the reclassification study in spring 2015, when this Duarte variant was reclassified. The reclassification study sample size was determined by the number of people who received the Duarte variant result. Following institutional review board approval, a genetic counselor (K.L.L.) called the 33 cases who had previously learned their Duarte variant carrier status. They were told, "Since the original report, our lab realized the change you were originally told about does not actually make someone a carrier for Duarte galactosemia. It is another variant in the same gene (GALT) that causes a person to be a carrier...we tested your sample to check whether you are truly a carrier of Duarte galactosemia." Participants who were still carriers $(n=23)$-positive testers -were told, "The test was positive, meaning that you have the second variant and are a carrier of Duarte galactosemia...the information you were originally told is still correct. Your

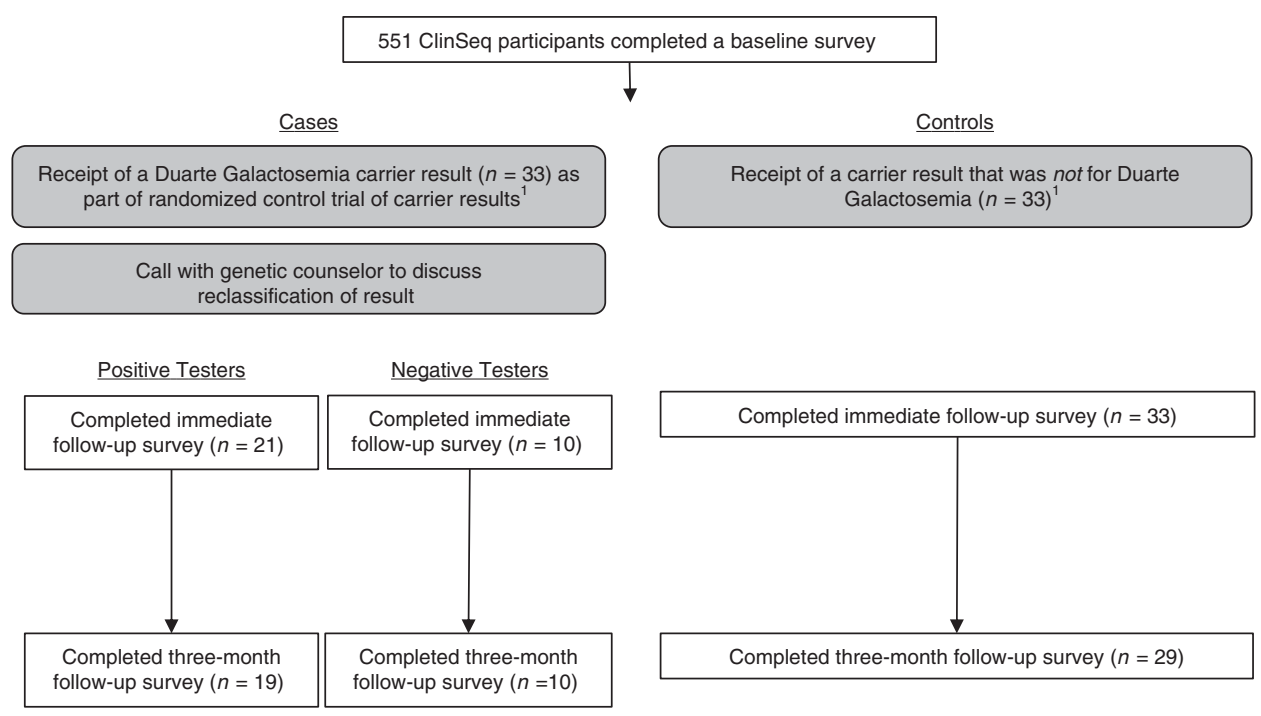

Figure 1 Study flow diagram and number of participants completing each assessment. ${ }^{1}$ Participants were randomized to receive these carrier results either from a genetic counselor or through a web platform. The results of this RCT are not analyzed or reported here. 


\section{ORIGINAL RESEARCH ARTICLE}

children/grandchildren are still at increased risk of Duarte galactosemia. There is a $0.14 \%$ risk to grandchildren, $0.28 \%$ risk to children, and $0.003 \%$ risk to the general population." Participants who were no longer believed to be carriers ( $n=10)$-negative testers - were told, "The test was negative, meaning that you do not have the second variant and are not a carrier of Duarte galactosemia. The risk for your children/ grandchildren is the same as that of anyone in the general population (not zero)." For the reclassification study, 21 positive testers and 10 negative testers (94\%) completed an online or paper survey after learning about this change. This survey, administered shortly after the reclassification, is the "immediate follow-up survey." A second follow-up online or paper survey administered 3 months later was completed by 19 positive testers and 10 negative testers (88\%).

To determine whether high or low endorsement on a particular outcome, or changes over time, were a function of learning about a change in one's result, data were also collected from 33 controls who received carrier results through the RCT but no reclassification. Controls were matched to cases on the date of their original carrier result return (within 30 days of the return of results to a case) and on severity and age of onset for the results they received. Controls received carrier results for multiple conditions; myeloperoxidase deficiency and hemochromatosis were the most commonly returned results. Seven potential controls did not respond and were replaced by comparable participants who were responsive. For controls, the "immediate follow-up survey" occurred at the time of the reclassification for cases; controls did not receive new information at that time. Twenty-nine of the 33 controls (88\%) who completed the immediate follow-up completed the 3-month survey. Data were analyzed from 58 participants who completed all three assessments (baseline, immediate, and at 3 months).

\section{Measures}

We asked three open-ended questions: (i) in your most recent meeting with the genetic counselor, what did she tell you about your genetic result?; (ii) what is your response to learning this information? In other words, how did learning this information make you feel and what did learning this information make you think?; and (iii) what are your thoughts about genetic information about health in general? Only cases (not controls) completed Questions 1 and 2 due to a programming oversight.

Of the following quantitative measures, only intentions to share results and perceived ambiguity were asked at baseline (completed shortly after enrollment in the parent ClinSeq study) and at the immediate and 3-month follow-ups of both cases and controls.

Intentions to share results were assessed at all three time points ( $1=$ "extremely unlikely" to $7=$ "extremely likely"). At baseline, participants were asked, "How likely is it that you will share your result(s) with your family members?" At follow-up assessments, cases reported about the change in their result. Perceived ambiguity was included to assess participants' direct reactions to learning of a reclassification of their genome result, and was assessed as the average of five statements (Cronbach's $\alpha_{\text {baseline }}=0.837, \alpha_{\text {immediate }}=$ $\left.0.676, \alpha_{3 \text {-month }}=0.769\right):^{12}$

"I don't believe my sequencing will be trustworthy."

"I don't believe my sequencing results will be accurate."

"I don't think my sequencing results will give clear answers about my future health."

"It seems like my sequencing results will be interpreted in many different ways."

"I think scientists won't be able to interpret much of my sequencing results."

At the immediate and 3-month follow-up assessments, the perceived ambiguity items were modified to refer to the results participants had already received (e.g., "I think scientists are not able to interpret much of my sequencing results.") Items were accompanied by a 5-point Likert scale ( 1 = "strongly disagree" to $5=$ "strongly agree").

The following five measures were assessed only at the immediate and 3-month follow-up time points. Intentions to learn genetic test results for preventable and unpreventable disease were assessed with two individual items: "How likely is it that you would choose to learn about a gene variant that predisposes you to a disease that can be prevented or treated in the future?" ( $1=$ "definitely no" to $5=$ "definitely yes"). The second item substituted "cannot" for "can." Because intentions to learn results were assessed on a different scale at baseline, changes were assessed only from the immediate to the 3-month follow-up. We assessed intentions to learn additional results because it was feasible (albeit unlikely) that participants who received a reclassification might subsequently perceive genomic information as being less valuable, which could be manifested in decreased interest in this type of information.

Participants rated perceived utility with, "My results can help me to better understand my genetic make-up" ( 1 = "not confident" to 5 = "very confident"). Negative emotions were assessed as the average of three items from the MICRA Questionnaire $^{13}$ assessing the extent to which participants felt upset, anxious or nervous, or regretful about their results $\left(0=\right.$ "never" to $3=$ "often"; $\alpha_{\text {immediate }}=0.731, \alpha_{3 \text {-month }}=$ $0.900)$. We applied a square root transformation to negative emotions (Table 1 footnote). Owing to an error, only cases reported perceived accuracy of results: "How confident are you that the information you just received about your sequencing result is accurate?" ( $1=$ "not at all" to $5=$ "very confident"). To reduce skew and kurtosis, we applied a log transformation and reverse-scored these values (Table 1 footnote).

Sex and race were coded as female/male and nonwhite/ white, respectively. We also examined age in years at the time of the baseline survey, years of education (high school, some college or technical school, college graduate, postgraduate), average household income $(<\$ 25,000 /$ year, $\$ 25,000$ to $\$ 49,999, \$ 50,000$ to $\$ 74,999, \$ 75,000$ to $\$ 100,999$, 
$\stackrel{w}{\text { th }}$ Table 1 Main effects of group and time from repeated-measures ANOVAs and specific pairwise comparisons

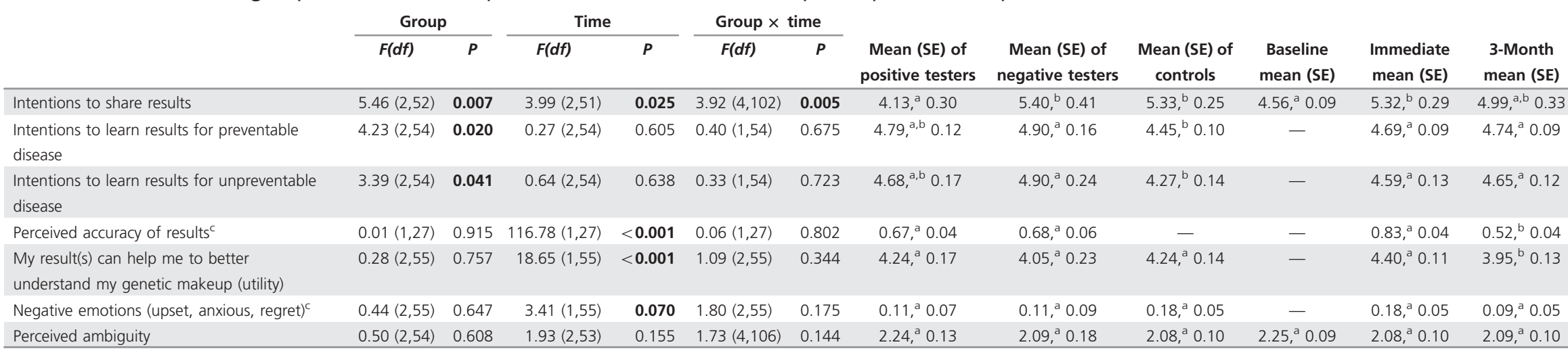

Statistics reported correspond with Wilks' lambda. Different superscripts indicate significant differences between participant groups or time points at $P<0.05$. $P$ values significant below $<0.10$ are shown in bold for ease of interpretation.

We examined homogeneity of variance across groups using SPSS Statistics 22. Mauchly's test of sphericity was nonsignificant for both measures assessed at all three assessments (perceived ambiguity and intentions to share results). Levene's test of inequality of error variances was significant $(P<0.10)$ for each measure at a minimum of one time point for four measures: (i) negative emotions, (ii) intentions to learn preventable and (iii) unpreventable disease results, and (iv) understanding. Because of unequal cell sizes (typically 19 positive testers, 10 negative testers, and 29 controls), we examined possible corrections (Tamhane, Dunnett T3, Games-Howell, Dunnett C) which provided adjusted $P$ values for the pairwise comparisons across groups. In all cases but one, the significance patterns (indicated by the alphabetical superscripts) were the same in the original results and application of any of the four possible corrections. For intentions to learn results for unpreventable diseases, means between positive testers and controls differed significantly in the uncorrected data but not when applying corrections. In the text, we present $P$ values for pairwise comparisons among groups based on the conservative Tamhane's correction for intentions to learn results for preventable and unpreventable disease.

${ }^{\top}$ Mean values are from transformed scales. For negative emotions: original skew: 2.40 , original kurtosis: 4.65 ; transformed skew: 2.02 , transformed kurtosis: 2.50 . For perceived accuracy: original skew: -1.82 , original kurtosis: 4.63 ; transformed skew: -0.53 , transformed kurtosis: -0.31 .

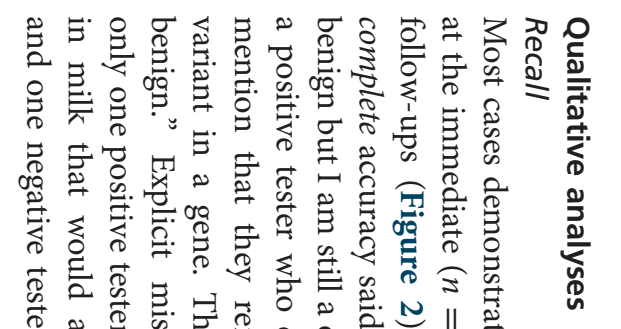

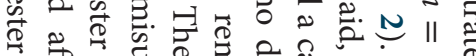

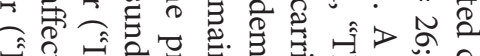
₹

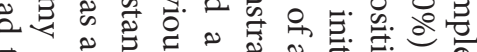

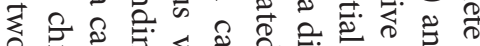

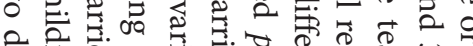

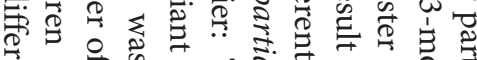

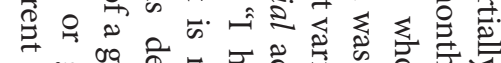

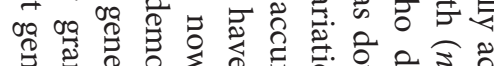
क्ञ

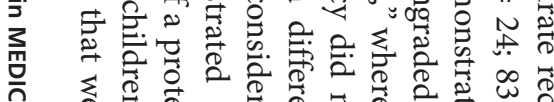

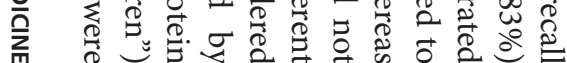

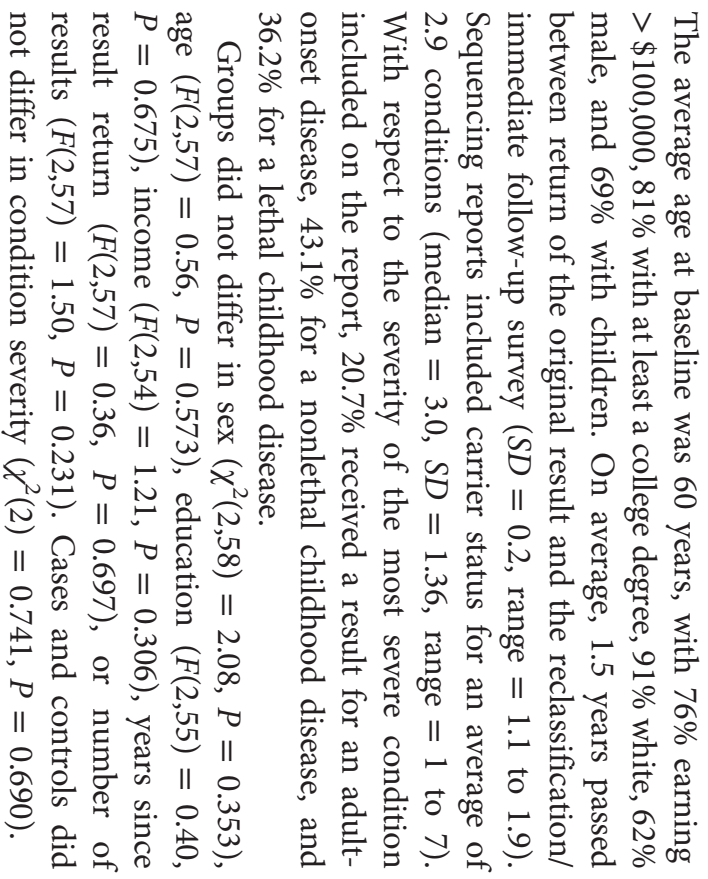

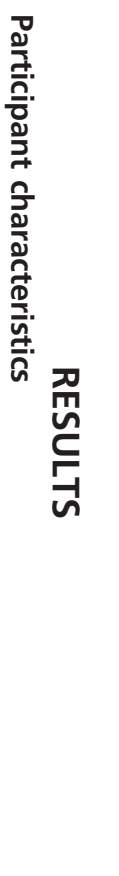

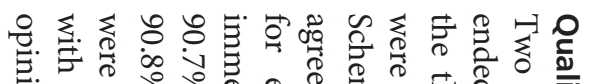

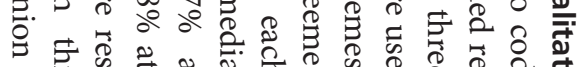

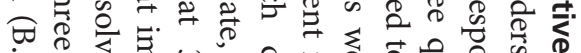

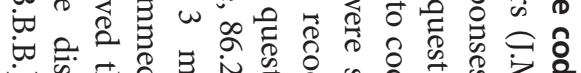

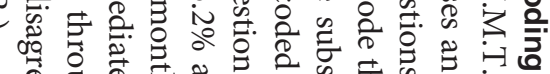

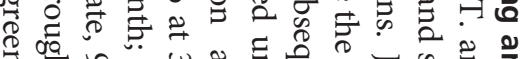
定

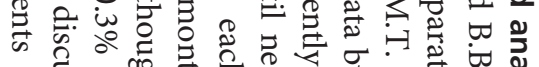

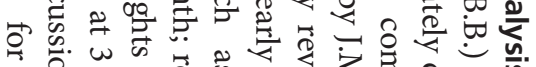

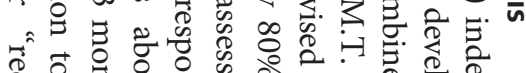

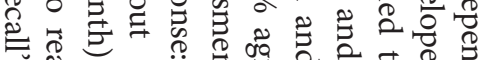

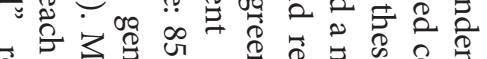

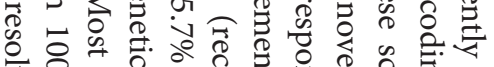

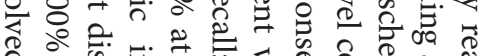

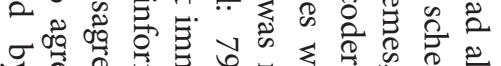

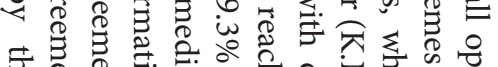

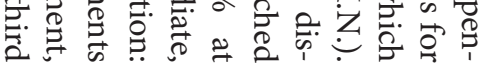

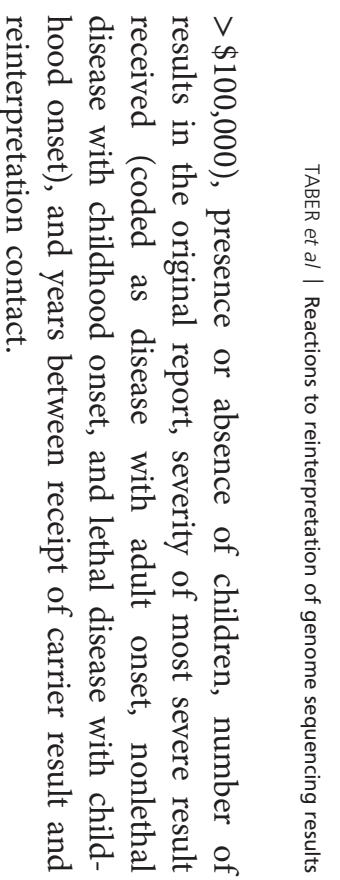



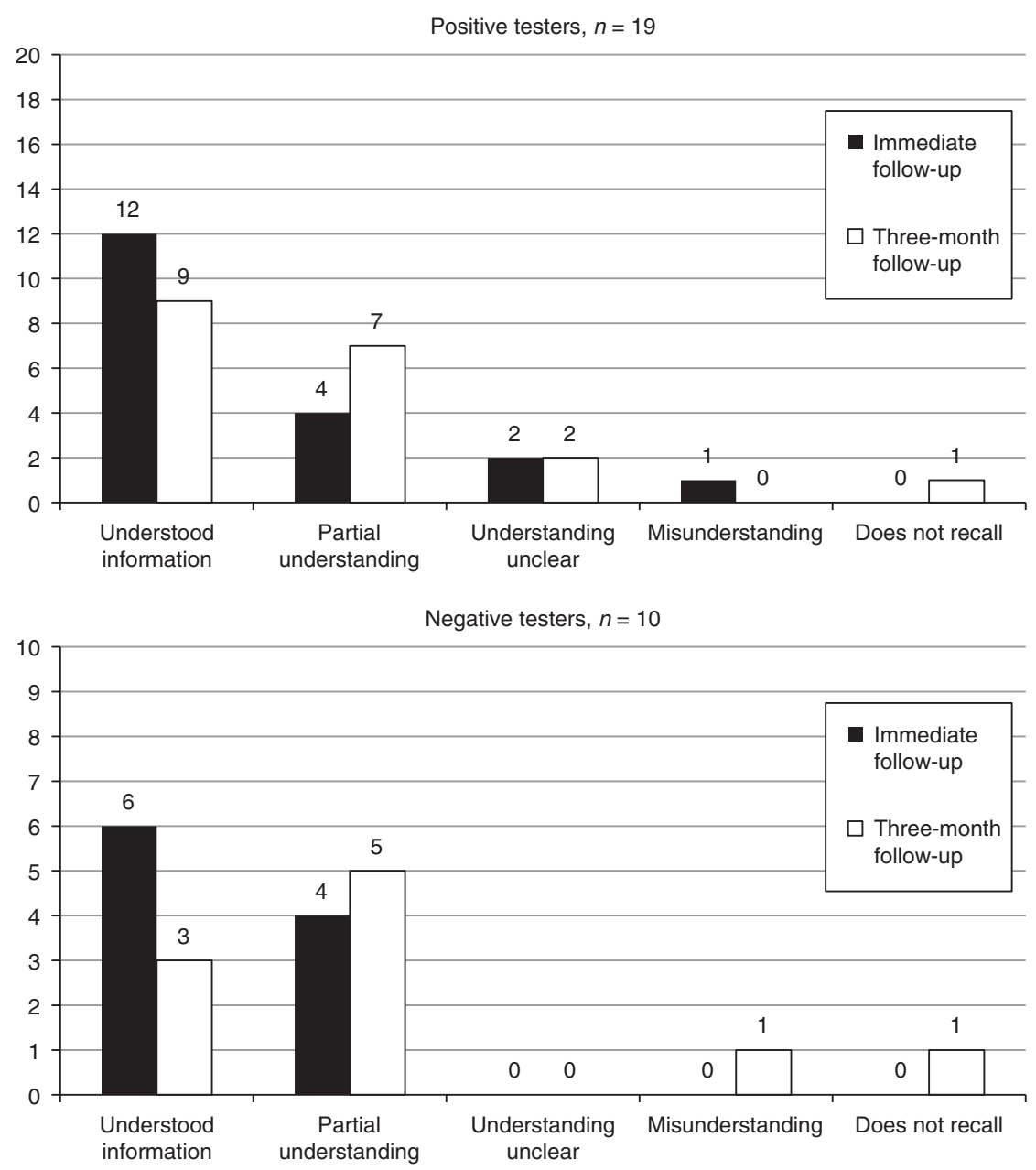

Figure 2 Accuracy of recall of information among positive and negative testers at the immediate and 3-month follow-ups. Bar labels indicate number of respondents who gave a particular response. Understood information: if positive tester, variant interpretation changed and implications remain the same; if negative tester, variant interpretation changed and no longer a carrier or gene is benign. Partial understanding: mentions a change to genetic result but not specifying the nature of the change or implications of the result; if negative tester, may say that implications have changed but not specify in what way. Understanding unclear: no evidence that participant understood revised results; no response. Misunderstanding: may have misunderstood revised results.

associated with some abnormalities, but neither was very serious and they would not affect me, but maybe our children/grandchildren, etc. None of these conditions have been experienced by [our children or grandchildren]"). Understanding of four cases was indeterminate ("Yes," "Yes, quite thoroughly," "I had no markers indicating any suggestion of a problem," and "Slight increase in one genetic risk, slight decrease in another") at the immediate or 3-month follow-up. Although these four cases demonstrated indeterminate understanding at one of the two assessments, three of these four cases demonstrated accurate recall at the assessment at which they did not have indeterminate understanding.

\section{Reactions}

When asked how the information made them think or feel (Figure 3), neutral reactions were common (positive testers: 68\% at immediate and 3 months; negative testers: $40 \%$ at immediate, $60 \%$ at 3 months). Neutral responses included the absence of a negative emotional response ("No big deal," "I feel the same as before") and statements that health implications were minimal or nonexistent ("I have very little to worry about in terms of disease proclivity carried in my genes"). Positive reactions were more common among negative testers ( $60 \%$ at immediate, $40 \%$ at 3 months; "I felt very comfortable and relieved with the results") than positive testers (26\% at immediate, $16 \%$ at 3 months; "I was glad to learn of this additional information"). Overall, $79 \%$ of cases $(n=23)$ expressed positive or neutral responses on both surveys. Some cases ( $21 \%$ at immediate, $24 \%$ at 3 months) made positive comments about ClinSeq ("It makes me feel that you are being diligent and continuing to massage the data," "Glad study is progressing with success") or commented on science more broadly ("Confirmed that we are still learning about...variation in the genome," "It shows that the field of medical genomics is still in its infancy"). Only one case, a 

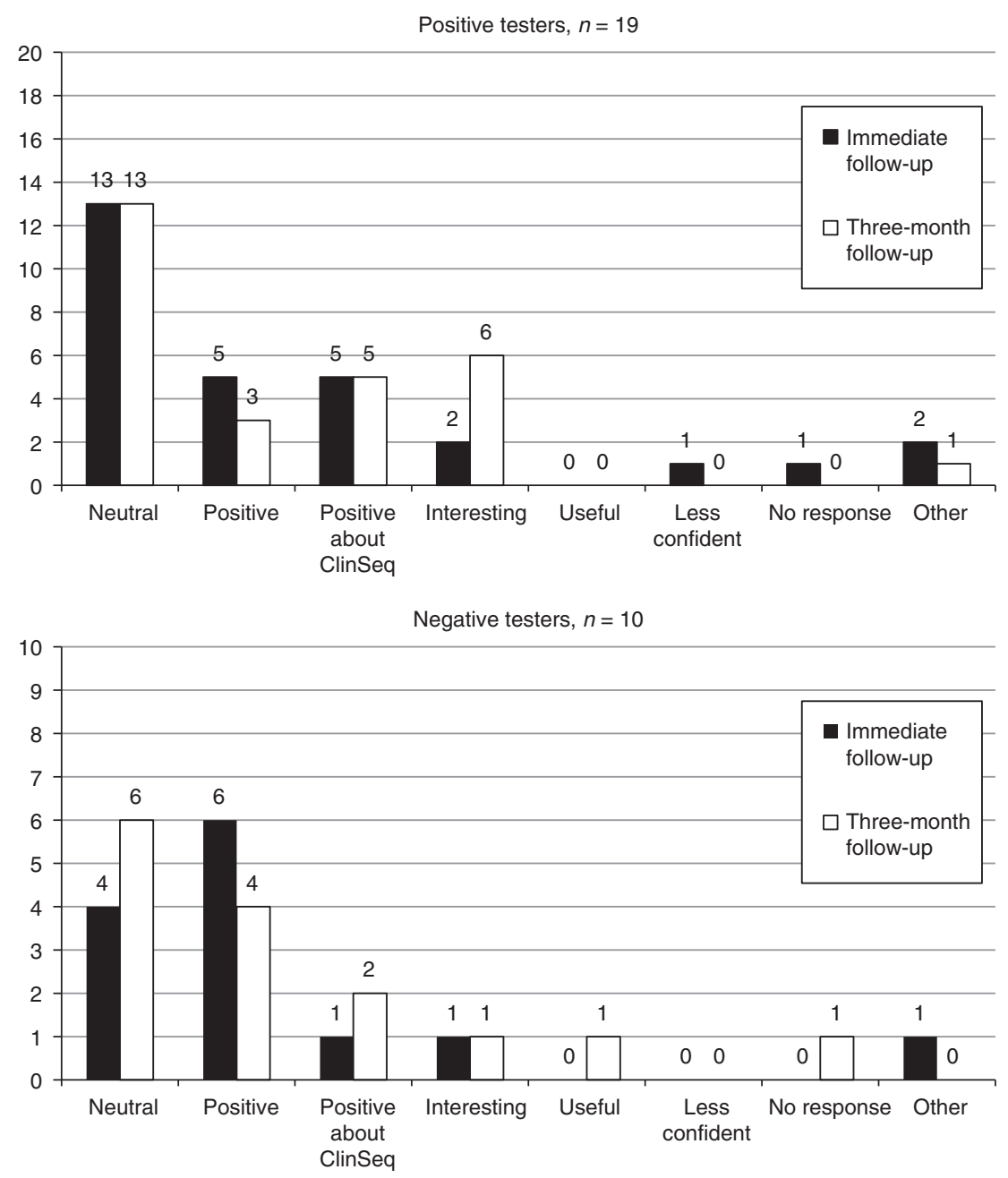

Figure 3 Nature of response to genetic information among positive and negative testers at the immediate and 3-month follow-ups. Bar labels indicate number of respondents who gave a particular response. Neutral: lack of a negative response, "feeling fine"; no change in emotional response; health implications are not serious/nonexistent or the change to the result is minor. Positive: reassured; information is good to know or good news. Positive about ClinSeq: positive comment about the ClinSeq study; good to know correction was found; glad to be updated; comment about the nature of science or genomic research. Interesting: information is interesting. Useful: information is useful or beneficial. Less confident: less confident in results. Other responses were "No opinion," "Curious about how the determination was done," "Pleased to be able to ask specific questions about the findings and get clarification of terms," "Want to leave to possibly help (inform) my children and grandchildren." Participants could give more than one response.

positive tester, had a negative reaction: "I felt less confident in the testing procedures and it makes me feel less sure of the reported results." Figure 3 shows additional responses.

\section{Thoughts about genetic information}

Nearly $80 \%$ of respondents expressed positive views about genetic information (Figure 4). Respondents stated that genetic information would be beneficial for oneself ("It's a good thing because it tells me what the future might hold for me"), one's family ("I would want to know as much as possible so...my children...can be informed and prepared"), or health in general ("Genetic information can be valuable to prevent or treat health issues"); expressed a desire for more information ("I'd like to know more about my genetic variants") or a belief that all information is beneficial ("This info can never do harm; knowledge is useful and good"); or made vague positive statements ("Helpful"). Other positive comments addressed the potential usefulness of genetic information in the future ("It has a great deal of promise, benefits to patients, benefits to medical research," "Genetic sequencing is a great opportunity").

Some respondents (from 10 to $37 \%$, depending on group and assessment) were uncertain of benefits from genetic information. These statements indicated indifference to genetic results ("Not really concerned. Had not thought about it since the last survey"), beliefs that benefits of genetic testing are not fully realized ("Genetic information is in its infancy and there are more developments to come"), or expressed limited usefulness or predictive ability of genetic information ("It can be useful in a limited number of cases; more data will help, but our genetic makeup can never accurately predict what ailments we'll encounter"). For some, 

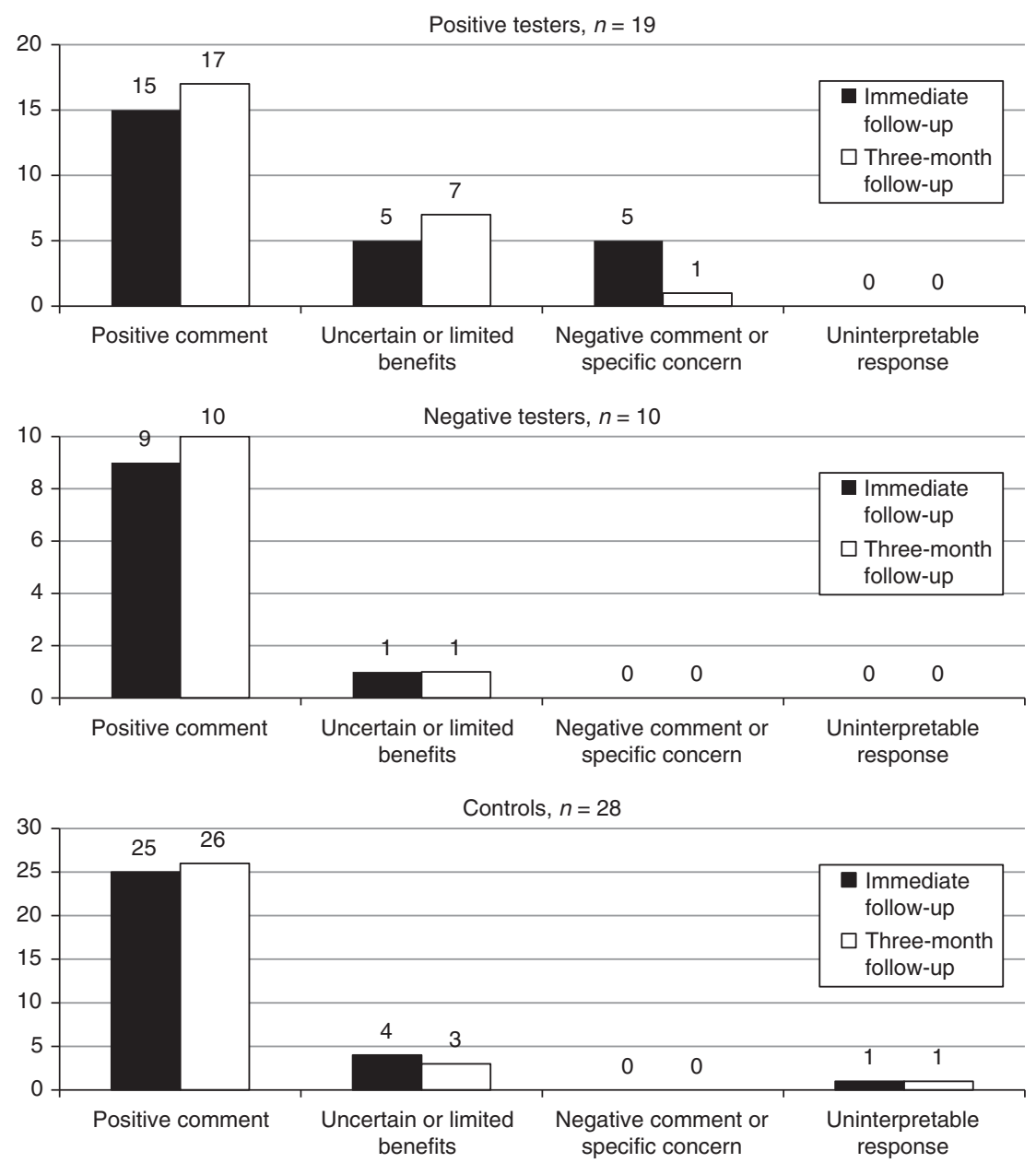

Figure 4 Thoughts about genetic information among cases and controls at the immediate and 3-month follow-ups as a function of testing status (positive versus negative). Bar labels indicate number of respondents that gave a particular response. Positive comment: perceives health implications or benefits for oneself or one's family or in general; general, vague positive comment; wants more information and believes all information is useful to have; thinks genetic information will be more helpful or continue to be helpful in the future. Uncertain: is uncertain of benefits; perceives limited benefit; unsure whether genetic information is useful; thinks genetic information has limited predictive ability (including statements that other factors are also important for health); thinks results will not have an impact on health or is unconcerned about results. Negative: makes negative comment or raises specific concern about genetic testing. Irrelevant or uninterpretable: response is irrelevant to the question or uninterpretable; no thoughts.

these statements were accompanied by positive comments ("Helpful to learn about these things, but don't feel they have much impact on my health").

Only five participants, all positive testers, expressed negative beliefs or specific concerns about genetic information at either assessment. Concerns were about financial cost, that negative information might be depressing, and that "ethical and moral boundaries must be maintained." One positive tester questioned whether insurance companies should be given genetic information at both assessments and said, "The ability to change genetic codes to eliminate some serious diseases is a capability that can be used for irksome or evil purposes." Only one of these five positive testers-who stated that: "The process of testing for alterations is based on statistical inference and all results have an associated variance. These results lower my confidence in what is being reported"-did not also provide a response that was coded as a positive response.

\section{Quantitative analyses}

We conducted repeated-measures ANOVA testing for changes in beliefs over time (baseline, immediate, at 3 months) as a function of participant group (positive testers, negative testers, controls). Tests are two-sided; no adjustments for multiple comparisons were made. Table 1 includes main effect and interaction statistics, means for each group collapsed across time, and means at each assessment collapsed across group. Time since result, and number of conditions reported, were considered but not included as covariates because neither variable was significantly correlated with any dependent variable at more than one assessment.

Intentions to share results changed differently over time as a function of group

There was a significant time $\times$ group interaction (Table 1) for intentions to share results with family members. Positive 
testers' intentions did not differ across assessments (all Ps $>0.162)$, with means around the midpoint of the scale ( $M=4.13$ out of 7 collapsed across time). Negative testers' intentions increased from baseline $(M=4.60)$ to the immediate follow-up $(M=6.00 ; P=0.019)$, but did not differ between baseline and 3 months $(M=5.60, P=0.141)$ or between the immediate and 3-month follow-ups $(P=0.567)$. Controls' intentions increased from baseline $(M=4.52)$ to the immediate $(M=6.00, P<0.001)$ and 3 month follow-ups $(M=5.48, \quad P=0.022)$. At baseline, intentions did not differ across groups (all Ps $>0.72$ ). At the immediate and 3-month follow-ups, however, positive testers had lower intentions than negative testers (immediate: $P=0.010$; 3-month: $P=0.057$ ) and controls (immediate: $P=0.001$; 3-month: $P=0.023$ ), with no differences between negative testers and controls (immediate: $P=1.00$; 3-month: $P=0.886$ ). Interestingly, positive testers' responses at the immediate and 3-month surveys showed bimodal distributions with as many respondents reporting "extremely unlikely" ( $n=$ six at immediate, five at 3 months) as "extremely likely" (six at immediate, five at 3 months), with few at the midpoint (none at immediate, two at 3 months). Bimodal distributions were not seen for negative testers or controls.

\section{Intentions to learn future results differed by group}

Two measures showed significant main effects of group (Table 1). Intentions to learn results for preventable and unpreventable disease were high, with average values typically above 4.5 on a 5 -point scale. Negative testers reported higher intentions than controls to learn results for preventable disease $(P=0.016)$, with no differences between positive and negative testers $(P=0.818)$ or positive testers and controls $(P=0.074)$. Similarly, for unpreventable diseases, negative testers reported higher intentions than controls $(P=0.006)$, with no differences between positive and negative testers $(P=0.610)$ or positive testers and controls $(P=0.023)$.

\section{Changes over time shown for perceived accuracy, utility, and negative emotions}

Perceived accuracy, which was asked only of cases at immediate and 3-month surveys $(n=29)$, decreased over time (Table 1). All untransformed means, however, were above 4.20 on the 5-point scale $(M=4.38$ at immediate, $M=4.28$ at 3-month surveys). Further, $66 \%$ of cases reported no change in perceived accuracy, $14 \%$ reported increases, and $21 \%$ reported decreases. Thus, only one-third of cases' beliefs changed over time.

Perceived utility of results significantly decreased over time ( $M=4.40$ at immediate, $M=3.95$ at 3 -month surveys). Further examination revealed that $41 \%$ of participants reported decreased beliefs, with 55\% reporting no change and 5.2\% (all controls) reporting increased beliefs.

Negative emotions tended to decrease over time (Table 1) and were infrequently endorsed. Only $21 \%$ of participants at the immediate and $14 \%$ at the 3-month follow-up surveys reported mean scores $>0$. Overall, $14 \%$ reported decreased negative emotions, whereas $10 \%$ reported increases. Most reported no change.

No changes over time or group differences in ambiguity There were no significant effects for perceived ambiguity (Table 1).

\section{DISCUSSION}

Results suggest that older, high-socioeconomic-status adults who receive reinterpretations of their carrier status with neutral or positive health implications report minimal adverse effects. Qualitative data indicated that most respondents accurately recalled the change in their result, reacted neutrally or positively to the change, and expressed positive attitudes toward genetic information more broadly. Participants reported relatively low levels of perceived ambiguity (a factor conducive to sharing genetic test results with others ${ }^{14}$ ), low levels of negative emotions, and high intentions to share the information with family and to learn results in the future.

Some results were unexpected. For example, control participants who did not receive a reclassification reported lower intentions to learn future results than did negative testers. Participants who received a reclassification may have remained engaged because of the new development. Additionally, participants who remained carriers reported lower intentions to share this information than did either negative testers or controls, perhaps because the change was not novel. Further, $21 \%$ of cases perceived lower accuracy of results over time, with $41 \%$ of participants reporting decreased belief in the utility of the tests, suggesting a need to explore these outcomes in other datasets. Because baseline data were unavailable for these measures, it is unknown whether the reclassification contributed to this finding. Additionally, the carrier results returned conferred low risk and were uninformative about carriers' own health.

The closest analog to reclassification of genetic test results may be patient attitudes about disclosing medical errors, although the reclassification in the present study was not an error. In general, patients want to know about even minor medical errors. ${ }^{15,16}$ Patients want emotional support when errors occur, ${ }^{15}$ and the opportunity to discuss the reclassification with a genetic counselor likely mitigated any potential negative outcomes. Little research, however, exists on consequences of and responses to medical errors. ${ }^{17}$

Several characteristics probably buffered against adverse outcomes. As shown by open-ended responses, the change in results would have little impact on participants' or their children's health. Some participants received neutral news that they remained carriers, and others received good news that they were no longer carriers. Additionally, Duarte galactosemia is mild in severity and has reduced penetrance, and the reclassification of this variant may have had a smaller impact than other carrier results participants received. Participants were older, well-educated, and likely low in medical mistrust. ${ }^{18}$ The generalizability of these results as 
genome sequencing becomes more accessible is unknown. Participants had previous encounters with ClinSeq team members, and qualitative responses suggested positive relationships with the team. Research is needed to explore responses to reclassifications involving medically actionable results or reclassification from noncarrier to carrier status.

Strengths of this study included the design, which allowed us to account for changes over time and to compare cases who received a reclassification with controls who did not. Limitations include the small sample size for the quantitative analyses and the sample characteristics. Compared to younger adults, our older sample, who had likely completed reproductive decision-making, may have perceived their initial and reinterpreted carrier results as less threatening. Further, cases had two contacts with the genetic counselor, whereas controls had only one, which possibly contributed to the lack of negative outcomes among cases and introduced bias. With an optimal design we would have reported data collected immediately after the return of the initial result, but this reclassification study was conceptualized after learning of the reclassification.

Many questions remain, including how to determine the cost and effectiveness of "genomic reevaluation." 1 The present study is an initial examination of how learning of a reinterpretation of carrier status results with the resultant conferring of neutral or good news impacts people, and provides preliminary evidence that this information can be returned-at least in a high-socioeconomic-status samplewithout adverse short-term consequences.

\section{ACKNOWLEDGMENTS}

The ClinSeq study is supported by the Intramural Research Program of the National Human Genome Research Institute, grants HG200359 08 and HG200387 03. We thank Kristy Nguyen for her assistance with the qualitative coding and Kristen Fishler for her assistance with collecting survey data.

\section{DISCLOSURE}

J.M.T., W.M.P.K., K.L.L., B.B.B., and J.J.J. declare that they have no conflict of interest. L.G.B. is an uncompensated consultant to the Illumina Corporation and receives royalties from the Genentech Corporation.

\section{REFERENCES}

1. Bowdin S, Gilbert A, Bedoukian E, et al. Recommendations for the integration of genomics into clinical practice. Genet Med 2016;18: 1075-1084.

2. Manolio TA, Chisholm RL, Ozenberger B, et al. Implementing genomic medicine in the clinic: the future is here. Genet Med 2013;15:258-267.

3. Lewis $C$, Skirton $H$, Jones $R$. Can we make assumptions about the psychosocial impact of living as a carrier based on studies assessing the effects of carrier testing? J Genet Couns. 2011;20:80-97.

4. Hamilton JG, Lobel M, Moyer A. Emotional distress following genetic testing for hereditary breast and ovarian cancer: a meta-analytic review. Health Psychol 2009;28:510-518.

5. Bollinger JM, Scott J, Dvoskin R, Kaufman D. Public preferences regarding the return of individual genetic research results: findings from a qualitative focus group study. Genet Med 2012;14:451-457.

6. Biesecker BB, Klein W, Lewis KL, et al. How do research participants perceive "uncertainty" in genome sequencing? Genet Med 2014;16: 977-980.

7. Biesecker LG, Mullikin JC, Facio FM, et al. The ClinSeq Project: piloting large-scale genome sequencing for research in genomic medicine. Genome Res 2009;19:1665-1674.

8. Lewis KL, Han PK, Hooker GW, et al . Characterizing participants in the ClinSeq genome sequencing cohort as early adopters of a new health technology. PLoS One 2015;10:e0132690.

9. Lewis KL, Hooker GW, Connors PD, et al. Participant use and communication of findings from exome sequencing: a mixed-methods study. Genet Med, 2015;18:577-583.

10. Berry GT. Galactosemia: when is it a newborn screening emergency? Mol Genet Metab. 2012;106:7-11.

11. Ficicioglu C, Thomas N, Yager C, et al. Duarte (DG) galactosemia: a pilot study of biochemical and neurodevelopmental assessment in children detected by newborn screening. Mol Genet Metab. 2008;95, 206-212.

12. Taber J, Klein W, Ferrer, R, et al. Perceived ambiguity as a barrier to intentions to learn genome sequencing results. J Behav Med. 2015;38: 715-726.

13. Cella D, Hughes C, Peterman A, et al. A brief assessment of concerns associated with genetic testing for cancer: the Multidimensional Impact of Cancer Risk Assessment (MICRA) questionnaire. Health Psychol 2002;21:564-572.

14. Taber JM, Chang CQ, Lam TK, et al. Prevalence and correlates of receiving and sharing high-penetrance cancer genetic test results: findings from the Health Information National Trends Survey. Public Health Genomics. 2015;18:67-77.

15. Gallagher, TH, Waterman AD, Ebers AG, et al. Patients' and physicians' attitudes regarding the disclosure of medical errors. JAMA 2003;289: $1001-1007$

16. Witman AB, Park DM, Hardin SB. How do patients want physicians to handle mistakes?: A survey of internal medicine patients in an academic setting. Arch Int Med. 1996;156:2565-2569.

17. Mazor KM, Simon SR, Gurwitz JH. Communicating with patients about medical errors: a review of the literature. Arch Int Med. 2004;164: 1690-1697.

18. LaVeist, TA, Isaac, LA, Williams, KP. Mistrust of health care organizations is associated with underutilization of health services. Health Serv Res. 2009;44:2093-2105. 